\title{
Frecuencia de patologías de tejidos duros y blandos de la cavidad bucal y peribucal en niños de la región de los Esteros del lberá.
}

Frequency of diseases in hard and soft

tissue in the oral and circumoral cavity

in children of the region of the Iberá.
Frequência das doenças dos tecidos duros e moles da cavidade oral e da região perioral em crianças de lberá.
Fecha de Recepción

10 de abril de 2013
Aceptado para su publicación

28 de junio de 2013
María Cristina Ojeda

Subdirectora. Prof. Titular, Cátedra de Odontología Social y Comunitaria.

María Florencia Maldonado Seniquel Becaria de Ciencia y Técnica.

Roque Oscar Rosende

Director.

Lugar de Trabajo

Facultad de Odontología.

Avda Libertad 5450. Tel./fax: 3794457992

Código postal: 3400

\section{Resumen}

El presente trabajo refiere sobre resultados parciales del estado de la dentición y de los tejidos blandos y zonas involucradas de la cavidad bucal, en 300 niños de 5 y 12 años, concurrentes a 13 escuelas rurales de pueblos y parajes de los Esteros del lberá.

El estudio de estas patologías son dos de las dimensiones de análisis en la investigación del componente bucal de la salud del hombre del lberá.

El objetivo es disponer de información fidedigna que permita tomar decisiones, operativizar acciones de acuerdo a los recursos disponibles, y luego medir el impacto de las metas propuestas.

Debido a que la población objeto de estudio es de alta vulnerabilidad biológica y social, las jornadas comprenden actividades de investigación, extensión y docencia en servicio, respetando los principios adicionales de la bioética.

\section{Palabras Claves}

Epidemiología, Caries Dental, Lesiones Estomatológicas, Poblaciones de Riesgo.

\section{Summary}

This paper reports the partial results about the state of dentition, soft tissues and involved areas of the oral cavity in 300 children between 5 to 12 years, attending in 13 schools in a rural towns and area of lberá.

The study of these pathologies includes two 
dimensions of a global analysis in the investigation of the oral component of human health in the lberá.

The principal aim is to provide reliable information to make decisions, effectuate actions according through available resources, and then measure the impact of goals.

Because the study population is very vulnerable, both biologically and socially, the conferences include research, extension and education service, respecting the principles of bioethics.

\section{Key words}

Epidemiology, dental caries, stomatologic injury, risk populations.

\section{Resumo}

Este artigo relata sobre os resultados parciais do estado dos tecidos dentição e macio e áreas envolvidas da cavidade oral em 300 crianças, 5 aos 12 anos, atendendo 13 escolas em cidades rurais e locais das lberá. $O$ estudo destas patologias são duas dimensões de análise na investigação do componente oral de lbera a saúde humana. O objetivo é fornecer informações confiáveis para tomar decisões, ações de operacionalização de acordo com os recursos disponíveis, e, em seguida, medir o impacto das metas propostas Porque a população do estudo é alta vulnerabilidade biológica e social, a conferência incluem pesquisa, extensão e ensino em serviço, respeitando os princípios adicionais de bioética.

\section{Palavras chave}

Epidemiologia da cárie dentária, lesões estomatologista, populações de risco.

\section{Introducción}

La epidemiología desde las diferentes perspectivas se define como el estudio descriptivo, analítico o experimental de todo fenómeno socio-biológico que afecte a la población, así como de sus determinantes. Dispone de estrategias y recursos requeridos para focalizar campos de los fenómenos colectivos, tanto para la explicación de su etiología, como en la búsqueda de métodos de intervención más eficaces. Implica la construcción que aborda lo colectivo desde la dimensión socio-histórica de los procesos de enfermar y sanar'.

La caries se ha considerado como la enfermedad de mayor peso en la historia de la morbilidad bucal a nivel mundial. En la actualidad, su distribución y severidad varían de una región a otra y su aparición se asocia con factores socioculturales, económicos, del ambiente y del comportamiento. Su prevalencia afecta entre $60 \%$ y $90 \%$, tanto de la población infantil como de la adulta ${ }^{2}$ y es una latente preocupación para los profesionales sanitaristas ${ }^{3}$.

Aparece en la infancia con la erupción de los dientes temporarios, $y$ cuando no es tratada con medios preventivos y curativos sigue propagándose y causando la destrucción parcial o total de los mismos, y con ello afectándose la calidad de vida de las personas. Se espera que el odontólogo realice acciones de promoción y prevención, y que las personas comprendan lo referente a su auto responsabilidad, tanto en los aspectos higiénicos como en los nutricionales ${ }^{4}$.

La prevalencia de caries observada en niños/as de 6 a II años de edad da cuenta del compromiso de la dentición primaria y permanente afectada. La magnitud de la enfermedad en dentición primaria se reduce a medida que aumenta la edad, debido a la exfoliación y recambio dentario, mientras que en la dentición permanente aumenta con la edad5 Así también, las lesiones estomatológicas son diversas patologías que se pueden presentar tanto en la mucosa oral, como en sus estructuras involucradas. Usualmente tienen diversos factores etiológicos y características clínicas diferentes. La frecuencia en nuestro medio no está determinada 6 .

Los estudios realizados de la prevalencia de la patología oral son realmente escasos, a diferencia de los epidemiológicos que versan sobre la caries y enfermedad periodontal. Diferentes investigadores han mostrado dispares apreciaciones y una gran variabilidad de casos en las prevalencias de las lesiones mucosas orales en distintas regiones del mundo ${ }^{7}$.

El alto grado de influencias del medio ambiente sobre los seres humanos, permiten afirmar que muchas de las enfermedades estomatológicas son resultado de ésta interacción, siendo los niños especialmente susceptibles ${ }^{8}$.

Para enfocar la solución de los problemas detec- 
tados, es necesario identificar las necesidades, las demandas, las expectativas sociales, como así también, los cambios en las respuestas sectoriales organizativas y en el financiamiento de la salud. Lo expresado, se considera muy importante para la región de Lagunas y Esteros del lberá, especialmente por su accesibilidad y sus características geográficas, la cual se describe como una depresión que comienza en la zona norte de la provincia de Corrientes, -departamento Ituzaingó- y se extiende en forma de arco hacia el suroeste a lo largo de $170 \mathrm{Km}$ y 16 a $40 \mathrm{Km}$ de ancho. El ingreso a sus pueblos y parajes es a través de rutas nacionales y provinciales de arena, tierra yo ripio, de difícil tránsito, sobre todo por las fuertes precipitaciones durante las estaciones de otoño y primavera'.

Es por ello, que a nivel epidemiológico, los pobladores del lberá son considerados de alto riesgo para las enfermedades bucales, debido a múltiples factores, entre los que se destacan, el poco acceso que tienen a los servicios de salud y a prácticas de higienes bucales inadecuadas, probablemente influidas por el contexto sociocultural en el cual están inmersos ${ }^{10}$.

\section{Material y Métodos}

Es un estudio epidemiológico transversal, observacional descriptivo.

La muestra actual se definió con 300 niños de ambos sexos, de 5 y 12 años de edad, concurrentes a 13 escuelas rurales de parajes del lberá dentro de los departamentos Gral. San Martín, Mercedes, San Miguel; Concepción; Ituzaingó; Santo Tomé y Virasoro. Se realizaron gestiones con Intendentes, Supervisoras de Escuelas y Guías de Turismo. Los datos se registraron en el formulario de evaluación de la salud bucodental, protocolizada por la OMS (1997). Sobre los tejidos duros del diente, se registró la situación de la corona y la raíz, como así también la necesidad de tratamientos, en dientes temporarios (5 años) y permanentes (I 2años).

En cuanto a los tejidos blandos, las lesiones se registraron según localización en la cavidad bucal y estructuras involucradas. En esta muestra se consideraron las lesiones elementales primarias, con registro fotográfico y descripción de las mismas.
A fin de relacionar los datos odontológicos con factores del medio ambiente social y biológico se realizaron entrevistas abiertas a informantes claves con registro magnetofónico y fichas temáticas.

\section{Resultados Parciales}

Se determinó una muestra por conveniencia de 150 niños de 6 años y 150 niños de 12 años.

\section{Estado de las piezas dentarias}

$n=150$ niños de 6 años: 60 niños (40\%) presentan piezas dentarias afectadas por caries dental con variados grados de severidad, en tanto que 90 niños (60\%) presentan piezas dentarias libres de caries.

\section{Gráfico $N^{\circ}$ I.}

ESTADO DE LAS PIEZAS DENTARIAS - 6 años -

\section{Q}

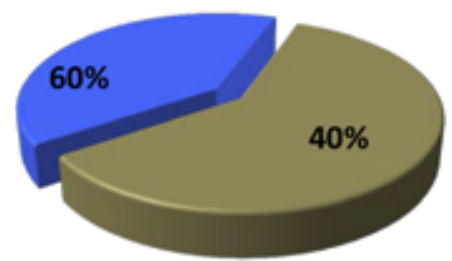

Por otro lado, la necesidad de tratamientos según severidad de las lesiones cariosas, se expresan en:

$40 \%$ de exodoncias

$30 \%$ de tratamientos pulpares

$30 \%$ obturaciones simples.

\section{NECESIDAD DE TRATAMIENTO - 6 años-}

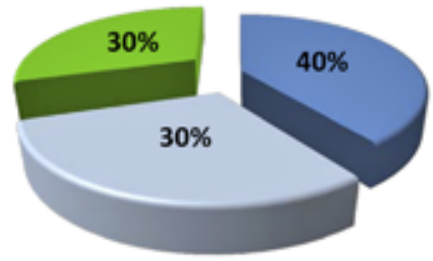

$$
\begin{aligned}
& \text { घ Exodoncias } \\
& \text { Tratamientos } \\
& \text { pulpares } \\
& \text { घobturaciones }
\end{aligned}
$$

$n=150$ niños 12 años: 40 niños (27\%), presentan piezas dentarias afectadas por caries dental con distintos grados de severidad, en tanto que 1 I 0 niños (73\%), con piezas dentarias libres de caries. 


\section{Gráfico $\mathbf{N}^{\circ} 3$}

ESTADO DE LAS PIEZAS DENTARIAS - 12 años-

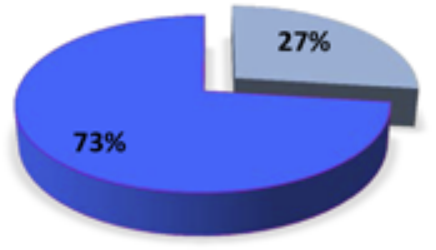

Afectadas por
caries

- Libre de caries

En cuanto a la necesidad de tratamientos según severidad de las lesiones cariosas, los datos que se registran son:

$30 \%$ exodoncias.

$10 \%$ tratamientos pulpares

$60 \%$ obturaciones simples.

\section{Gráfico $\mathbf{N}^{\circ} 4$}

\section{NECESIDAD DE TRATAMIENTO - 12 años-}

Exodoncias $\square$ Tratamiento pulpares [Obturaciones simples

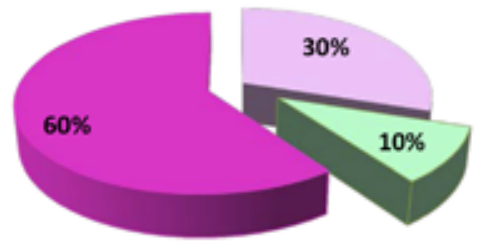

\section{Tejidos blandos de la cavidad bucal y zona peribucal}

Lesiones elementales primarias.

Pústulas $46 \%$

Vesículas $\quad 27 \%$

Erosiones $\quad 15 \%$

Grietas $7 \%$

Manchas

$$
5 \%
$$

Del total de la muestra de 300 niños, 98 presentaron lesiones (33\%), las cuales se describen como:

\section{Discusión}

En toda sociedad hay comunidades, familias y personas cuya probabilidad de enfermar es mayor que la de otros. Tales grupos son espe-

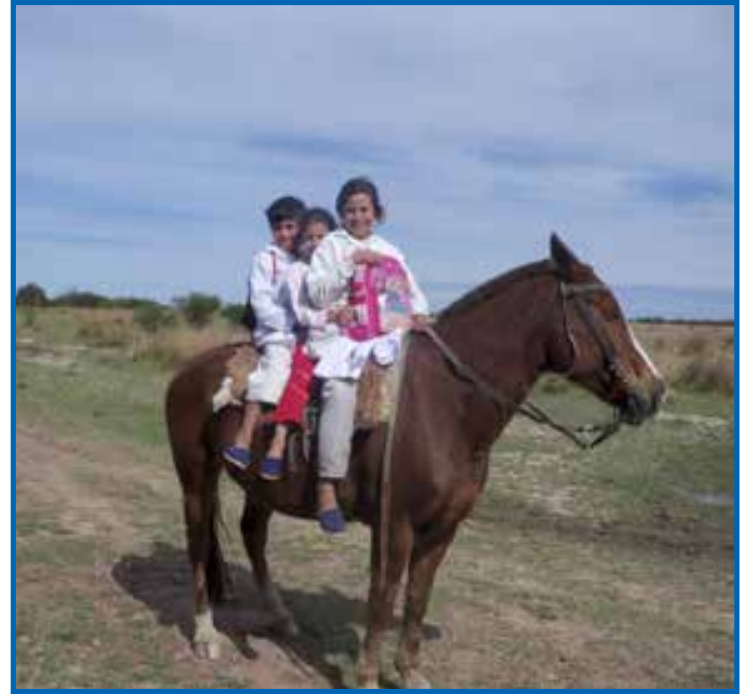

Niños de los Esteros.

cialmente vulnerables, y algunas de las razones pueden ser identificadas, coincidiendo con Matheu Genoveva, porque son el resultado de un número de características interactuantes, biológicas, ambientales, sociales y económicas.

Las patologías orales relevadas en los niños de 5 años demuestran alta frecuencia de caries dental con severidad de lesiones que indican un $70 \%$ entre exodoncias y tratamientos pulpares, mientras que a los 12 años disminuyen a un $40 \%$. Estos resultados coinciden con estudios realizados por Piovano Susana y colaboradores, como así también con los elementos de análisis para la situación de salud que propone la OMS. Se destaca que las piezas dentarias más afectadas en esta investigación son los primeros molares.

Por otro lado, las lesiones estomatológicas tienen también alta frecuencia en la región del lberá,

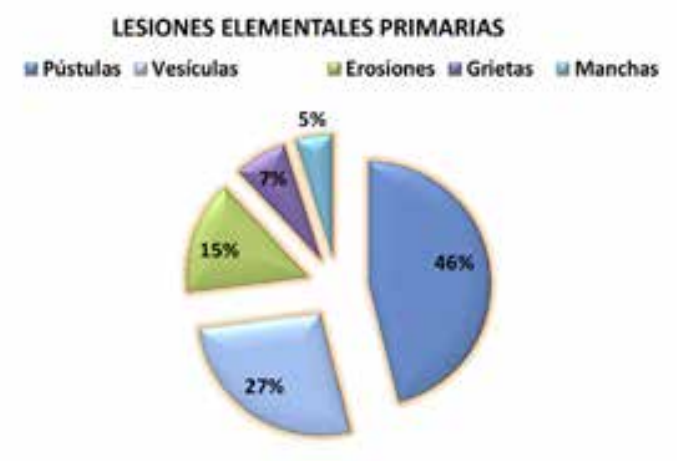




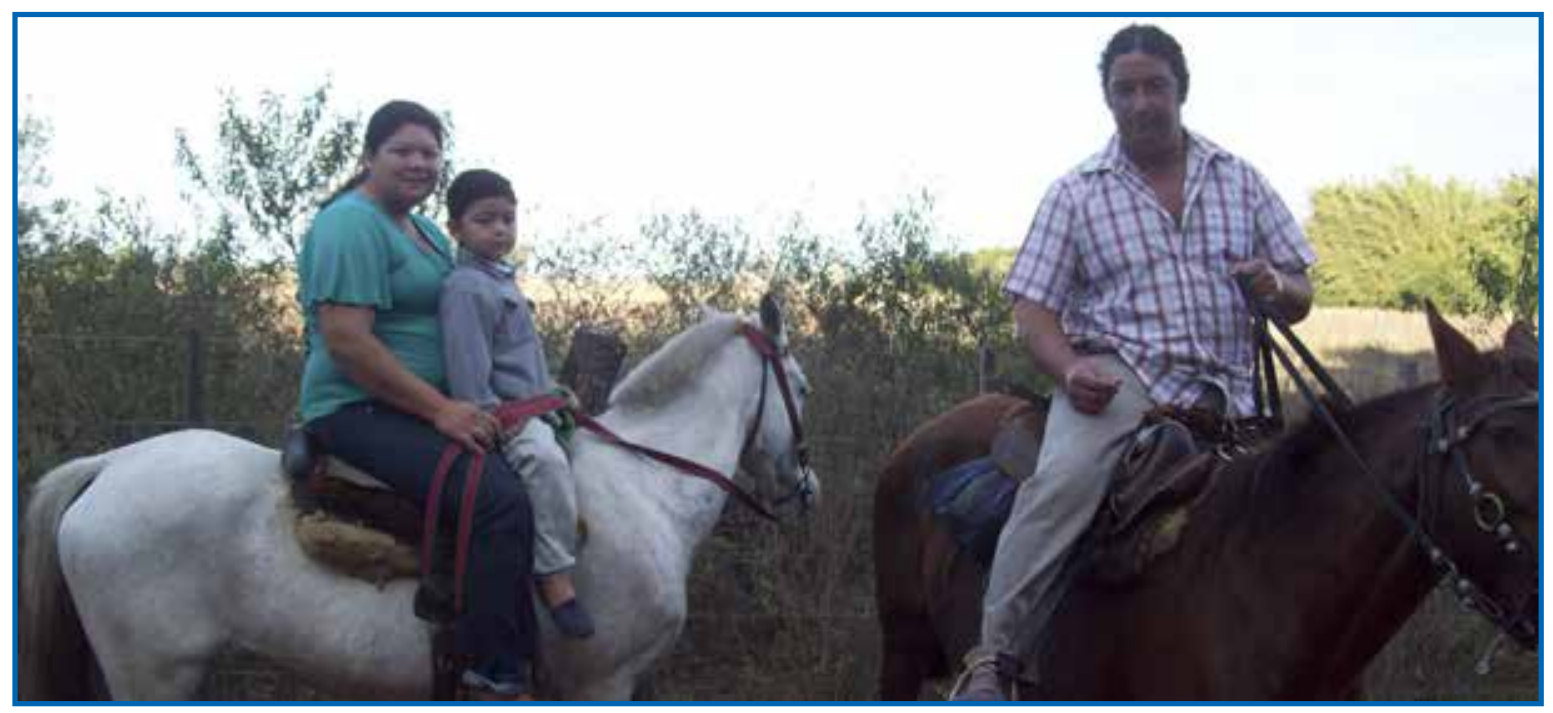

Familia Iberá.

algunas de ellas asociadas a infecciones en dientes temporarios no tratados. Su diagnóstico, según Kleiman, depende de la historia clínica, del conocimiento de las características específicas de cada una de ellas y de los factores de riesgo que las favorecen, entre ellas, falta de redes de drenaje, inadecuadas eliminación de residuos sólidos, consumo de agua de pozos, inaccesibilidad geográfica, acceso limitado a los servicios de salud y transporte, que retrasan la consulta preventiva y curativa. (Datos suministrados por informantes claves).

Respetando las recomendaciones del Consejo de Organizaciones Internacionales de Ciencias
Médicas (CIOMS) y la Organización Mundial de la Salud (OMS) para estudios epidemiológicos, paralelamente al registro de datos para la investigación, se brindó a la población, por medio de actividades de extensión, charlas de Educación para la Salud, atención clínica odontológica atraumáticas e información de interés a padres y docentes que participaron de las convocatorias. Lo anteriormente descrito se materializó en la integración de docencia en servicio, mediante la participación activa de profesores y educandos en el trabajo de campo, tanto en la identificación de los problemas de la comunidad, como en la solución de los mismos.

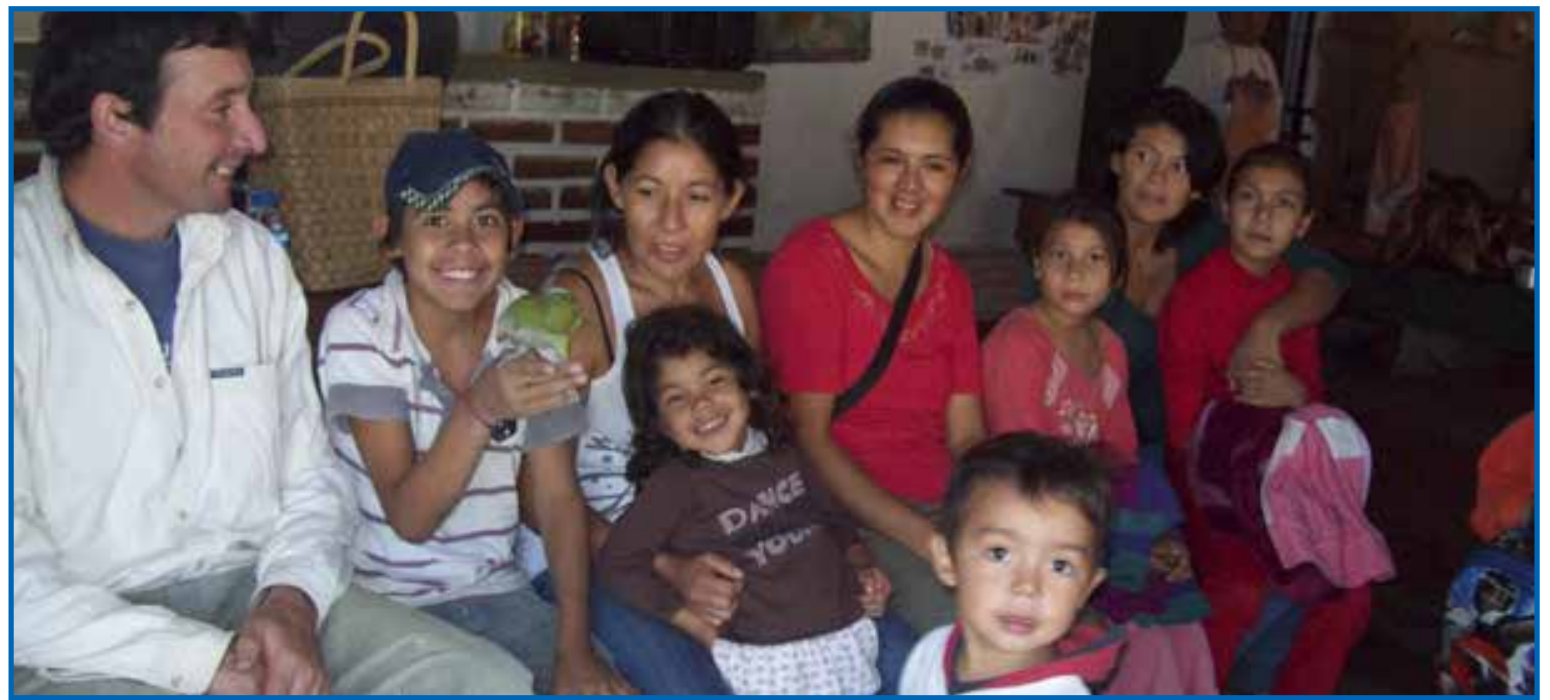

Convocatoria a las familias. 


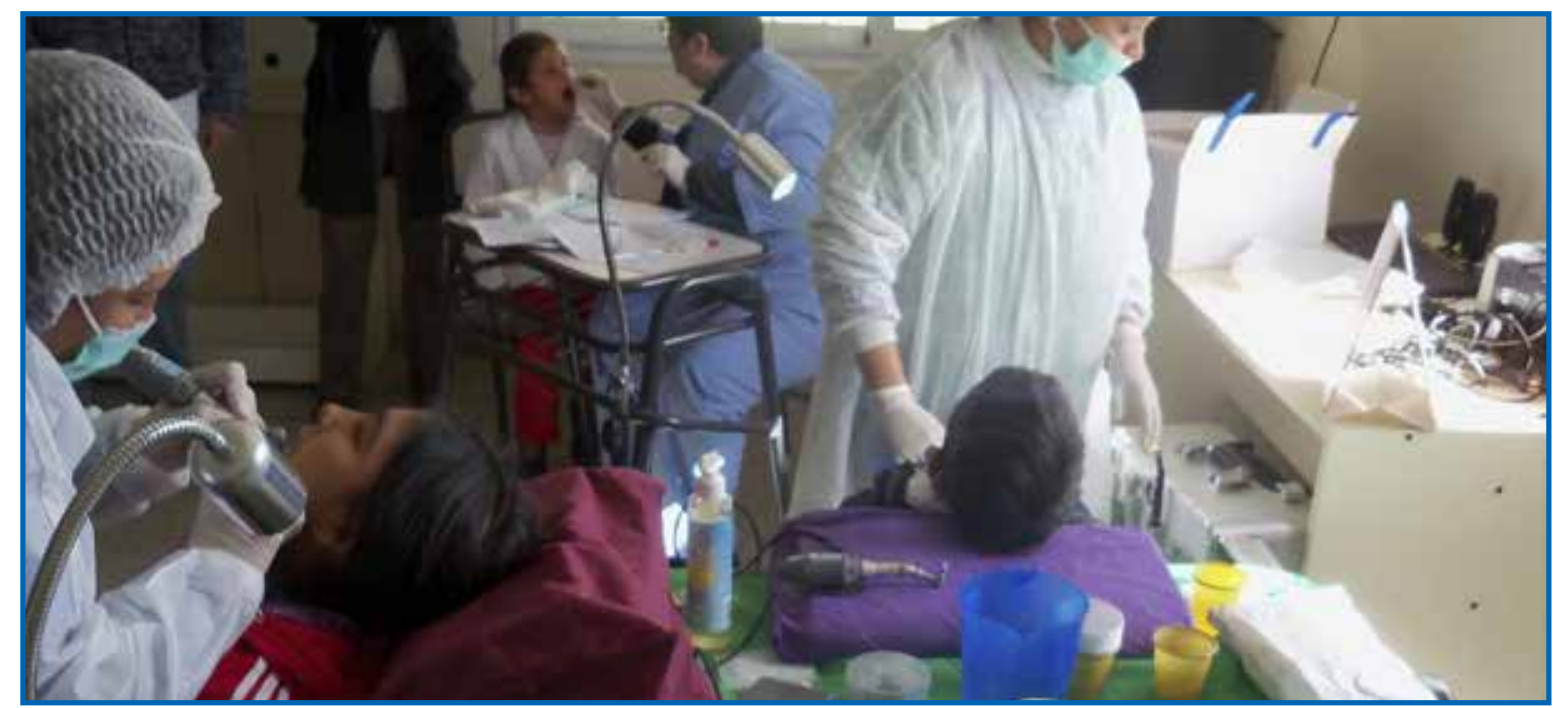

Movilidad de las familias en los parajes.

\section{Conclusión}

La caries dental y las enfermedades estomatológicas son patologías de alta frecuencia en la muestra seleccionada por conveniencia, en niños concurrentes a escuelas primarias de la región del lberá.

Las distancias geográficas, la falta de medios de transporte, las inclemencias del tiempo, la falta de servicios de salud, imposibilitan la asistencia odontológica preventiva y curativa.

La lesión elemental de mayor frecuencia es la pústula. La falta de agua potable y hábitos de higiene favorecen el desarrollo de infecciones asociadas en tejidos blandos de la cavidad bucal y zonas involucradas.

Algunas de ellas se presentan como consecuencias de abscesos dentarios no tratados en dientes temporarios.

El equipo de trabajo, integra investigación, docencia y extensión, considerando los principios adicionales de la bioética para investigaciones epidemiológicas, en un marco de respeto e intercambio de saberes.

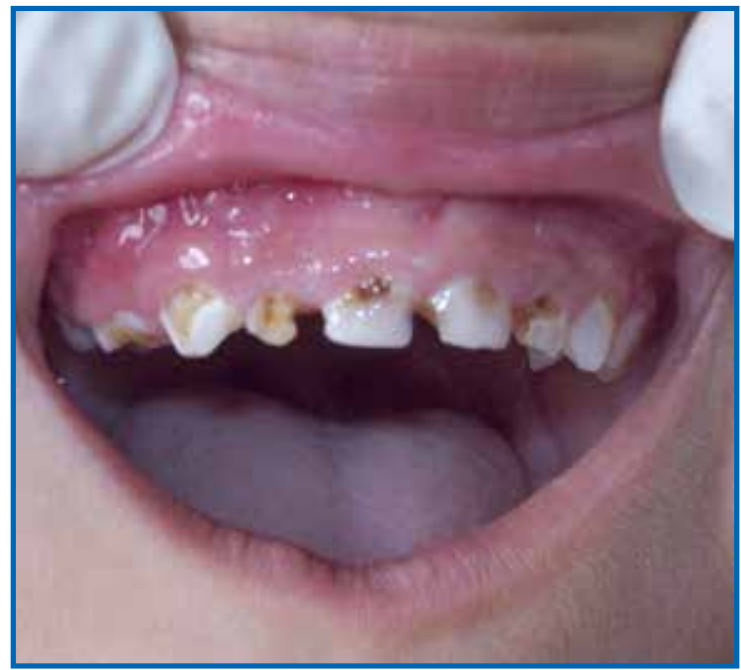

Caries en dentición temporaria.

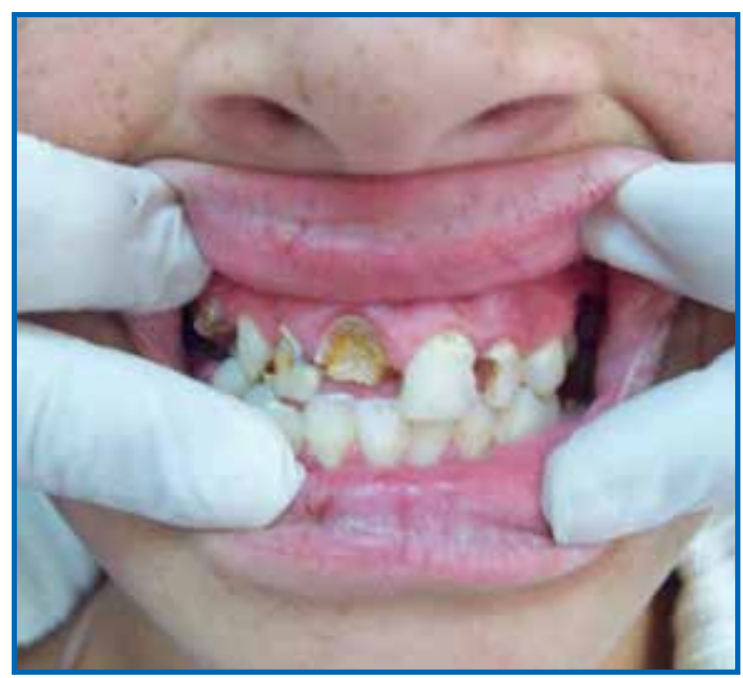

Caries en dentición permanente. 


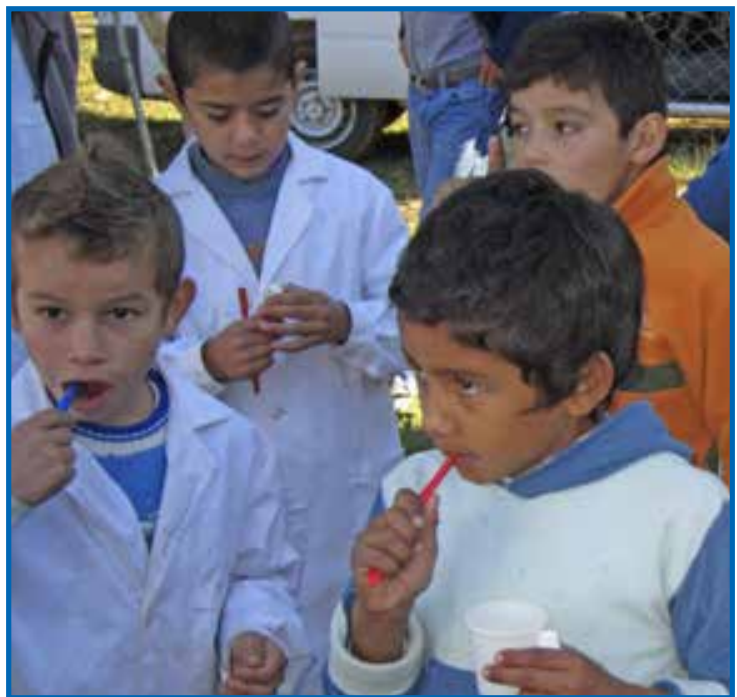

Enseñanza del cepillado.

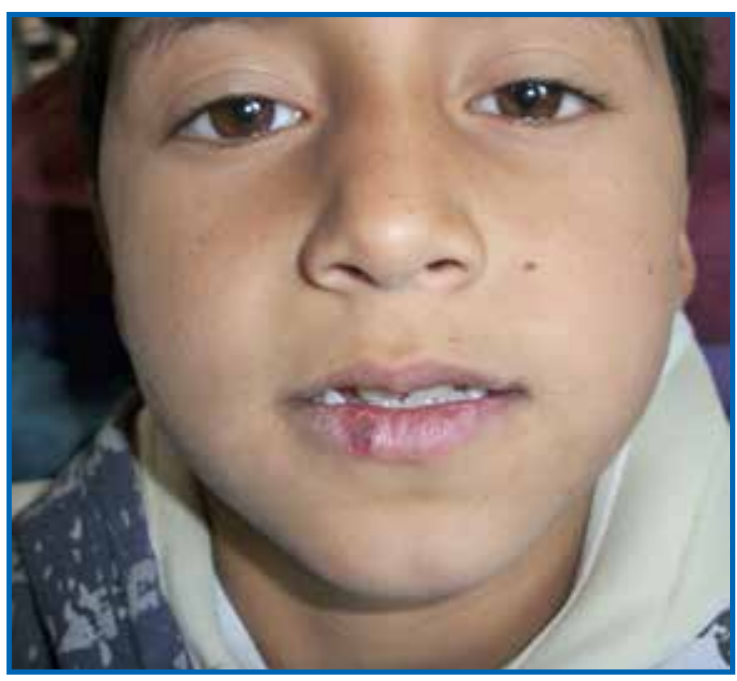

Erosión

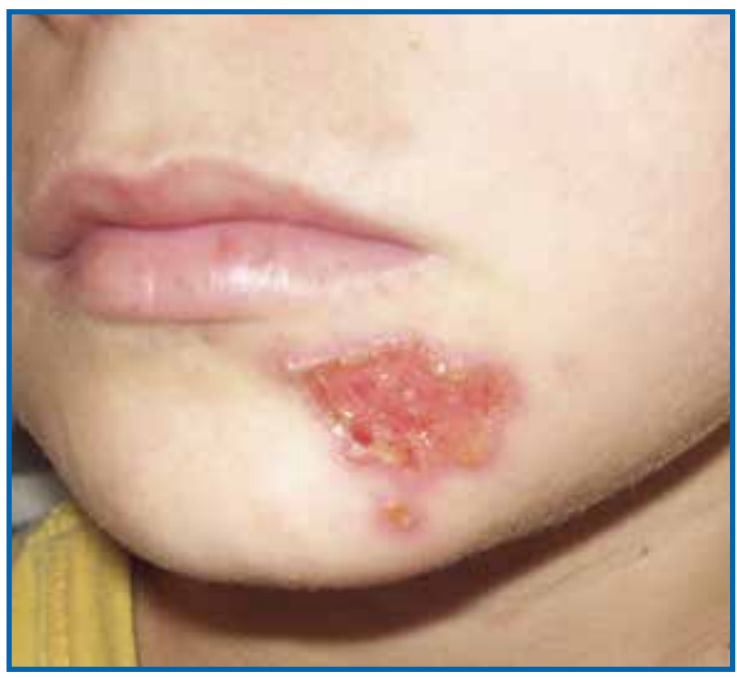

Vesículas

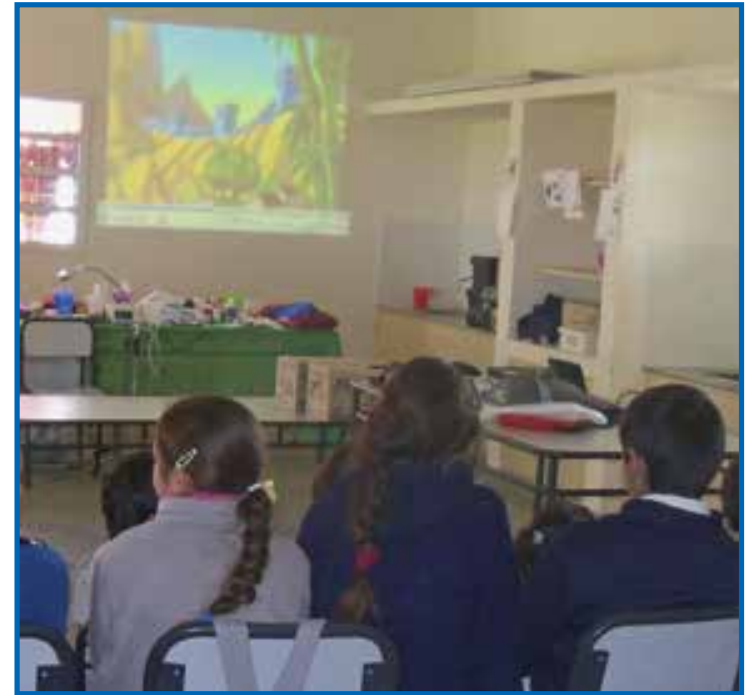

Proyección de películas.

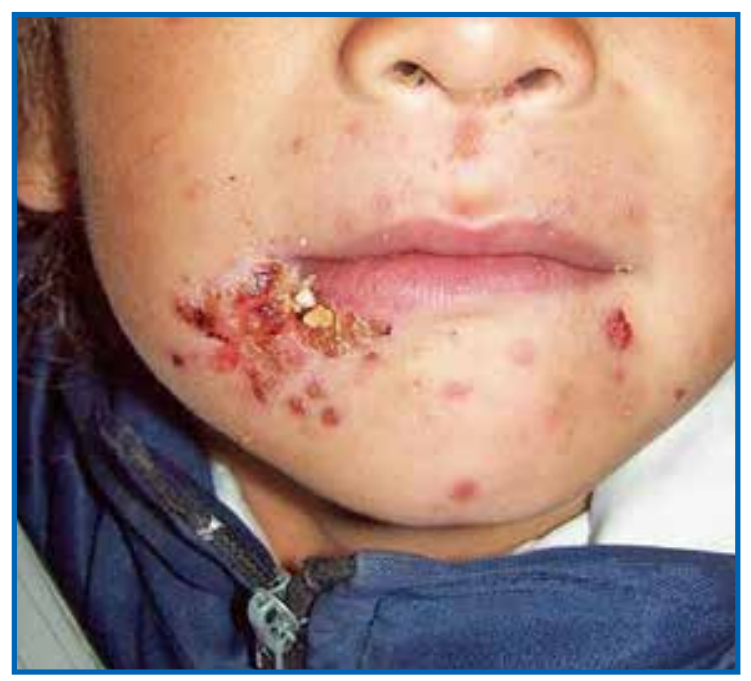

Pústulas.

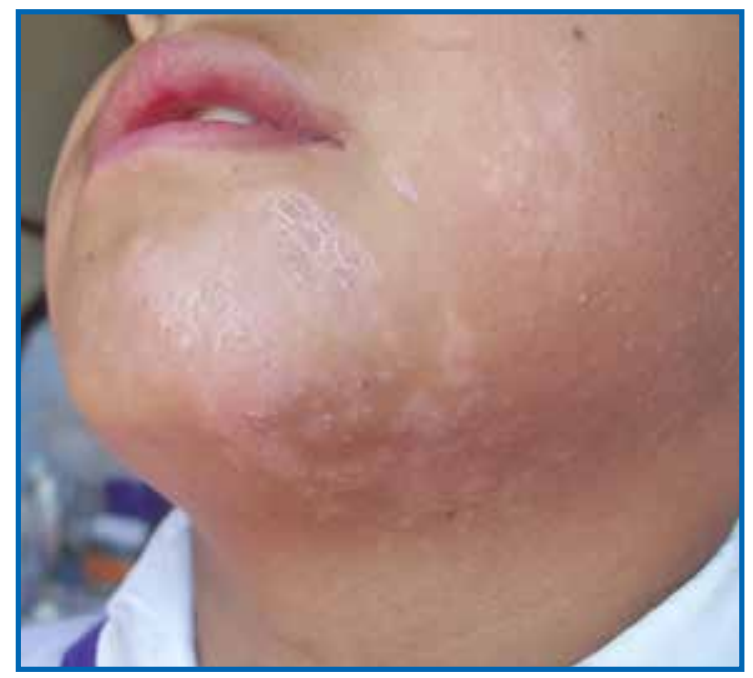

Manchas. 


\section{Referencias Bibliográficas}

I. OPS. Indicadores de Salud: Elementos básicos para el análisis de la situación de salud. Boletín Epidemiológico, 22 (4): Diciembre 2001.

2. Organización Mundial de la Salud. Investigaciones de salud oral básica. Métodos básicos. Ginebra: Organización Mundial de la Salud; 1987.

4. Pérez Luyo AG. ¿Es la caries dental una enfermedad infecciosa y transmisible?. Rev Estomatol Herediana. 2009; 19 (2): II8-124.

5. Cereceda María A; Simone Faleiros C; Ormeño Andrea C. Prevalencia de Caries en Alumnos de Educación Básica y su Asociación con el estado nutricional. Rev Chil Pediatr 20I0. [online] 8I (I): 28-36. Disponible en: http://www.scielo.cl/scielo. php?pid=S0370- Acceso 25/04/2013

6. Piovano Susana; Bordoni Noemí; Doño Raquel. Estado Dentario en Niños,Adolescentes y Adultos de la Ciudad Autónoma de Buenos Aires.

7. Revista de la Facultad de Odontología (UBA) Año 2008 [online]. Vol. 23. N 54/55. Disponible en: http://www.odon.uba.ar/ revista/2008vol23num5455/docs/piovano.pdf. Acceso 29/04/0I3.

8. Rioboo R. Estudio epidemiológico de las enfermedades y alteraciones bucodentales: patrones cambiantes y tendencias. Epidemiología de las lesiones de los tejidos blandos. En: Rioboo R. Odontología preventiva y odontología comunitaria. Madrid: Ed Avances; 2002. p. II 27-54.

9. Kleiman DV, Swango PA, PndborgJJ. Epidemiology of mucosal lesions in United States school children: 1986-1987. Community Dent Oral Epidemiol. 1994; 22: 243-53.

10. García-Pola MJ, García JM, González M. Estudio epidemiológico de la patología de la mucosa oral en la población infantil de 6 años de Oviedo (España). Medicina Oral 2002; 7: 184-9|.

II. Mahieu Genoveva "Propuesta Programa: Sistema de información y alerta sobre el cambio climático y vulnerabilidad de las poblaciones aledañas a los humedales de MERCOSUR caso piloto: esteros del Iberá. Provincia de Corrientes, Argentina. Proyecto PUND.Foixt /Reader 23 "programa de las Naciones Unidas para el desarrollo. Argentina. Arg. 2002/G35 (PRMS 1687).

12. Fernández González C, Núñez Franz L, Díaz Sanzana N. Determinantes de Salud oral en Población de 12 años. Rev. Clin. Periodoncia Implantol. Rehabil. Oral Vol. 4 (3); I I7-I2I, 20 I I.

13. Crivelli MR, Aguas S, Quarrancino C, Bazerque P. Influence of the socioeconomic estatus on oral mucosa lesion prevalence in schoolchildren. Community Dent Oral Epidemiol 1988; 16: 58-60.

14. Bruno Nifossi Prado, Shirley Trevisan, Dulce Helena Cabelho Passarelli. Estudo epidemiológico das lesões bucais no período de 05 anos epidemiological study of oral lesion in the period of 05 yearsrevista de odontologia da universidade cidade de são paulo 2010; 22 (I): 25-9, jan-abr.

15. Ramírez Puerta Blanca; Franco Cortéz Ángela. Caries en Dentición Permanente en Escolares de 8 a 12 y 15 años. Municipio de Frontino, Colombia. Revista Nacional de Odontología. 2010; 6 (II): 9-I5.

16. Organización Mundial de la Salud. Subsanar las desigualdades de una generación. Alcanzar la equidad sanitaria actuando sobre los determinantes sociales de la salud. Ginebra: Comisión sobre determinantes sociales de la salud. OMS; 2008.

17. Bessa C; Santos P; Aguilar M; Do Carmo M. Prevalence of oral mucosal alterations in children from 0 to 12 years old. J Oral Pathol Med 2004; 33: 17-32.

18. Olivero Rosa; Domínguez Antonio; Malpica Carmen. Principios bioéticos aplicados a la investigación epidemiológica. Acta Bioethica 2008; 14 (I). 